\title{
Augmented Lyapunov Method for BIBO Stabilization of Discrete System
}

\author{
Zixin Liu \\ School of Applied Mathematics, University of Electronic Science and Technology of China \\ No.4, Section 2, North Jianshe Road, Chengdu 610054, China \\ School of Mathematics and Statistics, Guizhou College of Finance and Economics \\ No.276, Luchongguan Road, Guiyang 55004, China \\ Tel: 86-851-8193-084Ｅ-mail: zixinliu@ foxmail.com
}

Shu Lv

School of Applied Mathematics, University of Electronic Science and Technology of China

No. 4, Section 2, North Jianshe Road, Chengdu 610054, China

Tel: 86-28-8320-2631Ｅ-mail: lvshu@uestc.edu.cn

Shouming Zhong

School of Applied Mathematics, University of Electronic Science and Technology of China

No. 4, Section 2, North Jianshe Road, Chengdu 610054, China

E-mail: zhongsm@uestc.edu.cn

The research is financed by the program for New Century Excellent Talents in University (NCET-06-0811) and the Research Fund for the Doctoral Program of Guizhou College of Finance and Economics (200702).

\begin{abstract}
The problem of bounded-input bounded-output (BIBO) stabilization for discrete-time uncertain system with time delay is investigated. By constructing an augmented Lyapunov function, some sufficient conditions guaranteeing BIBO stabilization and robust BIBO stabilization are established. These conditions are expressed in the forms of linear matrix inequalities (LMIs), whose feasibility can be easily checked by using Matlab LMI Toolbox. Two numerical examples are provided to demonstrate the effectiveness of the derived results.
\end{abstract}

Keywords: BIBO stabilization, Robust BIBO stabilization, Delay-dependent condition, Discrete-time system, Time delay

\section{Introduction}

Recently, in order to track out the reference input signal in real world, many researchers have focused their interest on the analysis of BIBO stabilization (see Guan et al. 1994, Xu and Zhong 1995, Wolfgang and Mecklenbräuker 1998, Michaletzky and Gerencser 2002, Partington and Bonnet 2004, Huang, Zeng and Zhong 2005). On the other hand, because of the finite switching speed, memory effects and so on, time delay is unavoidable in technology and nature. It can make the concerned control system become instable and oscillating, which cause the design and hardware implementation of the control system become difficult. Thus, BIBO stabilization analysis for delayed system is of great significance. In Li and Zhong (2008a), based on Riccati-equations, by constructing appropriate Lyapunov functions, some delay-independent BIBO stabilization criteria for a class of delayed control system with nonlinear perturbation were established. Based on Gronwall inequality, the problem of BIBO stabilization for system with multiple mixed delays and nonlinear perturbations were investigated in Li and Zhong (2008b). Similar to the method used in Li and Zhong (2008a), a class of linear delayed system with parameter uncertainty was considered in Li and Zhong (2007), and some robust BIBO stabilization criteria were derived in terms of linear matrix technique. In Li and Zhong (2009), the BIBO stabilization problem of a class of piecewise switched linear systems were further investigated. On the other hand, the problem of BIBO stabilization 
in mean square was also considered in Fu and Liao (2003). However, these previous results have been assumed to be in continuous time, but seldom in discrete time (see Bose and Chen 1995, Kotsios and Feely 1998). In practice, discrete-time control system is more applicable to problems that are inherently temporal in nature or related to biological realities. And it can ideally keep the dynamic characteristics, functional similarity, and even the physical or biological reality of the continuous-time systems under mild restriction. Thus, the BIBO stabilization analysis problems for discrete-time case are necessary.

Motivated by the above discussions, the main aim of this paper is to study the BIBO stabilization and robust BIBO stabilization problems for a class of discrete-time control system with time delay and parameter uncertainties. Based on linear matrix inequalities (LMIs) technique, an augmented Lyapunov function is constructed, and some sufficient conditions guaranteeing BIBO stabilization and robust BIBO stabilization are established. Finally, two numerical examples are provided to demonstrate the effectiveness of the derived results.

Notation: The notations are used in our paper except where otherwise specified. $\|\cdot\|$ denotes a vector or a matrix norm; $\mathbb{R}, \mathbb{R}^{n}$ are real and n-dimension real number sets, respectively; $\mathbb{N}^{+}$is positive integer set. $I$ is identity matrix; * represents the elements below the main diagonal of a symmetric block matrix; Real matrix $P>0(<0)$ denotes $P$ is a positive-definite (negative-definite) matrix; $\mathbb{N}[a, b]=\{a, a+1, \cdots, b\} ; \lambda_{\min }\left(\lambda_{\max }\right)$ denotes the minimum (maximum) eigenvalue of a real matrix.

\section{Preliminaries}

Consider the following discrete-time uncertain system with time delay described by

$$
\Sigma:\left\{\begin{array}{l}
x(k+1)=A(k) x(k)+B(k) x(k-\tau)+C(k) u(k), \quad k \in \mathbb{N}^{+} \\
y(k)=D(k) x(k) \\
x(k)=\varphi(k),-\tau \leq k \leq 0 .
\end{array}\right.
$$

where $x(k)=\left[x_{1}(k), x_{2}(k), \cdots, x_{n}(k)\right]^{T} \in \mathbb{R}^{n}$ denotes the state vector; $u(k)=\left[u_{1}(k), u_{2}(k), \cdots, u_{n}(k)\right]^{T} \in \mathbb{R}^{n}$ is the control input vector; $y(k)=\left[y_{1}(k), y_{2}(k), \cdots, y_{n}(k)\right]^{T} \in \mathbb{R}^{n}$ is the control output vector; Positive integer $\tau$ represents the transmission delay; $\varphi(\cdot)$ is vector-valued initial function and $\|\varphi\|_{\tau}$ is defined by $\|\varphi\|_{\tau}=\sup _{i \in \mathbb{N}[-\tau, 0]}\|x(i)\| ; A(k)=$ $A+\triangle A(k), B(k)=B+\triangle B(k), C(k)=C+\triangle C(k), D(k)=D+\triangle D(k) ; A, B, C, D \in \mathbb{R}^{n \times n}$ represent the weighting matrices; $\Delta A(k), \Delta B(k), \Delta C(k), \Delta D(k)$ denote the time-varying structured uncertainties which are of the following form:

$$
[\Delta A(k) \quad \Delta B(k) \quad \Delta C(k) \quad \Delta D(k)]=G F(k)\left[\begin{array}{llll}
E_{a} & E_{b} & E_{c} & E_{d}
\end{array}\right],
$$

where $G, E_{a}, E_{b}, E_{c}, E_{d}$ are known real constant matrices with appropriate dimensions; $F(k)$ is unknown time-varying matrix function satisfying $F^{T}(k) F(k) \leq I, \forall k \in \mathbb{N}^{+}$.

Let $u(k)$ be linear gain local state feedback with the reference input $r(k)$ for system $(1)$ as follows:

$$
u(k)=K x(k)+r(k)
$$

so as to ensure stabilization of the closed-loop delayed system.

To obtain our main results, we need introduce the following definitions and lemmas.

Definition 1 A real discrete-time vector $r(k) \in L_{\infty}^{n}$ if $\|r(k)\|_{\infty} \triangleq \sup _{k \in \mathbb{N}[0, \infty]}\|r(k)\|<+\infty$.

Definition 2 The control system (1) is said to be BIBO stabilized by the local control law (2) iffor every solution of system (1), $y(k)$ satisfies

$$
\|y(k)\| \leq \theta_{1}\|r(k)\|_{\infty}+\theta_{2}, k \in \mathbb{N}^{+} .
$$

where $\theta_{1}, \theta_{2}$ are known positive constants for every reference input $r(k) \in L_{\infty}^{n}$.

Lemma 1 (Lee and Radovic 1987) For any given vectors $v_{i} \in \mathbb{R}^{n}, i=1,2, \cdots, n$, the following inequality holds:

$$
\left[\sum_{i=1}^{n} v_{i}\right]^{T}\left[\sum_{i=1}^{n} v_{i}\right] \leq n \sum_{i=1}^{n} v_{i}^{T} v_{i} .
$$

Lemma 2 (Boyd et al. 1994) Given constant symmetric matrices $\Sigma_{1}, \Sigma_{2}, \Sigma_{3}$, where $\Sigma_{1}^{T}=\Sigma_{1}$ and $0<\Sigma_{2}=\Sigma_{2}^{T}$, then $\Sigma_{1}+\Sigma_{3}^{T} \Sigma_{2}^{-1} \Sigma_{3}<0$ if and only if

$$
\left(\begin{array}{cc}
\Sigma_{1} & \Sigma_{3}^{T} \\
\Sigma_{3} & -\Sigma_{2}
\end{array}\right)<0 \text { or }\left(\begin{array}{cc}
-\Sigma_{2} & \Sigma_{3} \\
\Sigma_{3}^{T} & \Sigma_{1}
\end{array}\right)<0
$$


Lemma 3 (Liu, Wang and Liu 2008) Let $N$ and E be real constant matrices with appropriate dimensions, matrix function $F(k)$ satisfies $F^{T}(k) F(k) \leq I$, then, for any $\epsilon>0, E F(k) N+N^{T} F^{T}(k) E^{T} \leq \epsilon^{-1} E E^{T}+\epsilon N N^{T}$.

Lemma 4 For any real vector $X, Y$ and positive-definite matrix $\Sigma>0$ with appropriate dimensions, it follows that

$$
2 X^{T} Y \leq X^{T} \Sigma X+Y^{T} \Sigma^{-1} Y \text {. }
$$

For designing the linear feedback control $u(k)=K x(k)+r(k)$ such that the closed-loop system (1) is BIBO stabilized by local control law (2), we first consider the nominal $\Sigma_{0}$ of $\Sigma$ defined by

$$
\Sigma_{0}:\left\{\begin{array}{l}
x(k+1)=A x(k)+B x(k-\tau)+C u(k), \quad k \in \mathbb{N}^{+} \\
y(k)=D x(k) \\
x(k)=\varphi(k),-\tau \leq k \leq 0 .
\end{array}\right.
$$

Substituting (2) into system (3) yields a closed-loop systems as follows:

$$
\Sigma_{0}:\left\{\begin{array}{l}
x(k+1)=(A+C K) x(k)+B x(k-\tau)+C r(k), \quad k \in \mathbb{N}^{+} \\
y(k)=D x(k) \\
x(k)=\varphi(k),-\tau \leq t \leq 0 .
\end{array}\right.
$$

Then, we can obtain the following BIBO stabilization results.

\section{Main results}

Theorem 1 For given positive integer $\tau>0$, local control law (2) with feedback gain matrix $K$ stabilizes the delayed system (4), if there exist positive-definite matrices $Q, R, H, P_{1}, P_{2}$, positive-definite diagonal matrix $Z$ with appropriate dimensions, such that the following LMI holds:

$$
\Xi_{1}=\left[\begin{array}{ccccc}
\Xi_{11} & \Xi_{12} & \Xi_{13} & \Xi_{14} & \Xi_{15} \\
* & \Xi_{22} & \Xi_{23} & \Xi_{24} & \Xi_{25} \\
* & * & \Xi_{33} & \Xi_{34} & \Xi_{35} \\
* & * & * & \Xi_{44} & \Xi_{45} \\
* & * & * & * & \Xi_{55}
\end{array}\right]<0
$$

where $Q=\left[\begin{array}{ccc}Q_{11} & Q_{12} & Q_{13} \\ * & Q_{22} & Q_{23} \\ * & * & Q_{33}\end{array}\right]>0$,

$\Xi_{11}=A^{T} Q_{11} A-Q_{11}+H+P_{1}+C K+K^{T} C^{T}, \Xi_{12}=A^{T} Q_{12} A-Q_{12}+B, \Xi_{13}=A^{T}\left(Q_{11}+Q_{13}\right)-I+K^{T} C^{T}, \Xi_{14}=$ $A^{T}\left(Q_{12}-Q_{13}\right)+B^{T}, \Xi_{15}=A^{T} Q_{13}-Q_{13}, \Xi_{22}=A^{T} Q_{22} A-Q_{22}-H, \Xi_{23}=A^{T}\left(Q_{12}^{T}+Q_{23}\right), \Xi_{24}=A^{T} Q_{22}-A^{T} Q_{23}$, $\Xi_{25}=A^{T} Q_{23}-Q_{23}, \Xi_{33}=Q_{11}+Q_{33}+Q_{13}+Q_{13}^{T}-2 I+P_{2}+\tau Z+R, \Xi_{34}=Q_{12}+Q_{23}^{T}-Q_{13}-Q_{33}, \Xi_{35}=Q_{13}+Q_{33}$, $\Xi_{44}=Q_{22}+Q_{33}-Q_{23}-Q_{23}^{T}-R, \Xi_{45}=Q_{23}-Q_{33}, \Xi_{55}=-\tau^{-1} Z$.

Proof. Constructing an augmented Lyapunov-Krasovskii function candidate as follows:

$$
V(k)=V_{1}(k)+V_{2}(k)+V_{3}(k),
$$

where $V_{1}(k)=\widetilde{X}^{T}(k) Q \widetilde{X}(k), \widetilde{X}^{T}(k)=\left[x^{T}(k), x^{T}(k-\tau), \sum_{i=k-\tau}^{k-1} \eta^{T}(i)\right], \eta(k)=x(k+1)-A x(k)$,

$$
V_{2}(k)=\sum_{i=k-\tau}^{k-1} x^{T}(i) H x(i)+\sum_{i=k-\tau}^{k-1} \eta^{T}(i) R \eta(i), \quad V_{3}(k)=\sum_{j=k-\tau}^{k-1} \sum_{i=j}^{k-1} \eta^{T}(i) Z \eta(i) .
$$

Set $X^{T}(k)=\left[x^{T}(k), x^{T}(k-\tau), \eta^{T}(k), \eta^{T}(k-\tau), \sum_{i=k-\tau}^{k-1} \eta^{T}(i)\right]$. Define $\Delta V(k)=V(k+1)-V(k)$, then along the solution of system (4) we can obtain that

$$
\begin{aligned}
\Delta V_{1}(k)= & \widetilde{X}^{T}(k+1) Q \widetilde{X}(k+1)-\widetilde{X}^{T}(k) Q \widetilde{X}(k) \\
= & x^{T}(k)\left[A^{T} Q_{11} A-Q_{11}\right] x(k)+2 x^{T}(k)\left[A^{T} Q_{12} A-Q_{12}\right] x(k-\tau)+2 x^{T}(k) A^{T}\left[Q_{11}+Q_{13}\right] \eta(k) \\
& +2 x^{T}(k) A^{T}\left[Q_{12}-Q_{13}\right] \eta(k-\tau)+2 x^{T}(k)\left[A^{T} Q_{13}-Q_{13}\right]\left(\sum_{i=k-\tau}^{k-1} \eta(i)\right)+x^{T}(k-\tau)\left[A^{T} Q_{22} A-Q_{22}\right] x(k-\tau) \\
& +2 x^{T}(k-\tau) A^{T}\left[Q_{12}^{T}+Q_{23}\right] \eta(k)+2 x^{T}(k-\tau) A^{T}\left[Q_{22}-Q_{23}\right] \eta(k-\tau)+2 x^{T}(k-\tau)\left[A^{T} Q_{23}\right. \\
& \left.-Q_{23}\right]\left(\sum_{i=k-\tau}^{k-1} \eta(i)\right)+\eta^{T}(k)\left[Q_{11}+Q_{33}+Q_{13}+Q_{13}^{T}\right] \eta(k)+2 \eta^{T}(k)\left[Q_{12}+Q_{23}^{T}-Q_{33}-Q_{13}\right] \eta(k-\tau)+2 \eta^{T}(k) \times \\
& {\left[Q_{13}+Q_{33}\right]\left(\sum_{i=k-\tau}^{k-1} \eta(i)\right)+\eta^{T}(k-\tau)\left[Q_{22}+Q_{33}-Q_{23}^{T}-Q_{23}\right] \eta(k-\tau)+2 \eta(k-\tau)\left[Q_{23}-Q_{33}\right]\left(\sum_{i=k-\tau}^{k-1} \eta(i)\right) . \quad(6) }
\end{aligned}
$$




$$
\Delta V_{2}(k)=x^{T}(k) H x(k)-x^{T}(k-\tau) H x(k-\tau)+\eta^{T}(k) R \eta(k)-\eta^{T}(k-\tau) R \eta(k-\tau) .
$$

From lemma 1, we have

$$
\begin{aligned}
\Delta V_{3}(k) & =\sum_{j=k+1-\tau}^{k} \sum_{i=j}^{k} \eta^{T}(i) Z \eta(i)-\sum_{j=k-\tau}^{k-1} \sum_{i=j}^{k-1} \eta^{T}(i) Z \eta(i) \\
& =\sum_{j=k-\tau}^{k-1} \sum_{i=j+1}^{k} \eta^{T}(i) Z \eta(i)-\sum_{j=k-\tau}^{k-1} \sum_{i=j}^{k-1} \eta^{T}(i) Z \eta(i) \\
& =\sum_{j=k-\tau}^{k-1}\left[\eta^{T}(k) Z \eta(k)-\eta^{T}(j) Z \eta(j)\right] \\
& =\tau \eta^{T}(k) Z \eta(k)-\sum_{i=k-\tau}^{k-1} \eta^{T}(i) Z \eta(i) \\
& =\tau \eta^{T}(k) Z \eta(k)-\sum_{i=k-\tau}^{k-1}(\sqrt{Z} \eta(i))^{T} \sqrt{Z} \eta(i) \\
& \leq \tau \eta^{T}(k) Z \eta(k)-\frac{1}{\tau}\left[\sum_{i=k-\tau}^{k-1} \eta(i)\right]^{T} Z\left[\sum_{i=k-\tau}^{k-1} \eta(i)\right] .
\end{aligned}
$$

On the other hand, by lemma 4 , for any positive-definite matrices $P_{1}, P_{2}$ with appropriate dimensions, we have

$$
\begin{aligned}
0 & =2 x^{T}(k)[C K x(k)+B x(k-\tau)+C r(k)-\eta(k)] \\
& =2 x^{T}(k) C K x(k)+2 x^{T}(k) B x(k-\tau)+2 x^{T}(k) C r(k)-2 x^{T}(k) \eta(k) \\
\leq & x^{T}(k)\left(C K+K^{T} C^{T}+P_{1}\right) x(k)+2 x^{T}(k) B x(k-\tau)-2 x^{T}(k) \eta(k)+r^{T}(k) C^{T} P_{1}^{-1} C r(k) \\
\leq & x^{T}(k)\left(C K+K^{T} C^{T}+P_{1}\right) x(k)+2 x^{T}(k) B x(k-\tau)-2 x^{T}(k) \eta(k)+\|r(k)\|^{2}\left\|C^{T} P_{1}^{-1} C\right\|, \\
0 & =2 \eta^{T}(k)[C K x(k)+B x(k-\tau)+C r(k)-\eta(k)] \\
& =2 \eta^{T}(k) C K x(k)+2 \eta^{T}(k) B x(k-\tau)+2 \eta^{T}(k) C r(k)-2 \eta^{T}(k) \eta(k) \\
& \leq 2 \eta^{T}(k) C K x(k)+2 \eta^{T}(k) B x(k-\tau)+\eta^{T}(k)\left(P_{2}-2 I\right) \eta(k)+r^{T}(k) C^{T} P_{2}^{-1} C r(k) \\
& \leq 2 \eta^{T}(k) C K x(k)+2 \eta^{T}(k) B x(k-\tau)+\eta^{T}(k)\left(P_{2}-2 I\right) \eta(k)+\|r(k)\|^{2}\left\|C^{T} P_{2}^{-1} C\right\| .
\end{aligned}
$$

Combining (6)-(10), we get

$$
\Delta V(k) \leq X^{T}(k) \Xi X(k)+\sigma\|r(k)\|^{2},
$$

where $\sigma=\left\|C^{T} P_{1}^{-1} C\right\|+\left\|C^{T} P_{2}^{-1} C\right\|$. If the LMI (5) holds, it follows that there exists a sufficient small positive scalar $\varepsilon>0$ such that

$$
\Delta V(k) \leq-\varepsilon\|x(k)\|^{2}+\sigma\|r(k)\|^{2} .
$$

On the other hand, it can easily to get that

$$
V(k) \leq \alpha_{1}\|x(k)\|^{2}+\alpha_{2} \sum_{i=k-\tau}^{k-1}\|x(i)\|^{2},
$$

where $\alpha_{1}=\lambda_{\max }(Q)[1+2 \tau]+\lambda_{\max }(R)+\tau \lambda_{\max }(Z), \alpha_{2}=2 \tau\left[\lambda_{\max }(Z)+1\right]+\lambda_{\max }(H)+2 \lambda_{\max }(R)$.

For any $\theta>1$, it follows from (13) that

$$
\begin{aligned}
\theta^{j+1} V(j+1)-\theta^{j} V(j) & =\theta^{j+1} \Delta V(j)+\theta^{j}(\theta-1) V(j) \\
& \leq \theta^{j}\left[\sigma \theta\|r(j)\|^{2}-\varepsilon \theta\|x(j)\|^{2}+(\theta-1) \alpha_{1}\|x(j)\|^{2}+(\theta-1) \alpha_{2} \sum_{i=j-\tau}^{j-1}\|x(i)\|^{2}\right] .
\end{aligned}
$$

Summing up both sides of (14) from 0 to $k-1$ we can obtain

$$
\begin{aligned}
\theta^{k} V(k)-V(0) & \leq\left[\alpha_{1}(\theta-1)-\varepsilon \theta\right] \sum_{j=0}^{k-1} \theta^{j}\|x(j)\|^{2}+\alpha_{2}(\theta-1) \sum_{j=0}^{k-1} \sum_{i=j-\tau}^{j-1} \theta^{j}\|x(i)\|^{2}+\sum_{j=0}^{k-1} \sigma \theta^{j+1}\|r(j)\|^{2} \\
& \leq \mu_{1}(\theta) \sup _{j \in \mathbb{N}[-\tau, 0]}\|x(j)\|^{2}+\mu_{2}(\theta) \sum_{j=0}^{k} \theta^{j}\|x(j)\|^{2}+\sum_{j=0}^{k-1} \sigma \theta^{j+1}\|r(j)\|^{2},
\end{aligned}
$$


where $\mu_{1}(\theta)=\alpha_{2}(\theta-1) \tau^{2} \theta^{\tau}, \mu_{2}(\theta)=\alpha_{2}(\theta-1) \tau \theta^{\tau}+\alpha_{1}(\theta-1)-\varepsilon \theta$. Since $\mu_{2}(1)=-\varepsilon \theta<0$, there must exist a positive $\theta_{0}>1$ such that $\mu_{2}\left(\theta_{0}\right)<0$. Then we have

$$
\begin{aligned}
V(k) & \leq \mu_{1}\left(\theta_{0}\right)\left(\frac{1}{\theta_{0}}\right)^{k} \sup _{j \in \mathbb{N}[-\tau, 0]}\|x(j)\|^{2}+\left(\frac{1}{\theta_{0}}\right)^{k} V(0)+\sigma \sum_{j=0}^{k-1} \frac{1}{\theta_{0}^{k-j-1}}\|r(j)\|^{2} \\
& \leq \mu_{1}\left(\theta_{0}\right)\left(\frac{1}{\theta_{0}}\right)^{k} \sup _{j \in \mathbb{N}[-\tau, 0]}\|x(j)\|^{2}+\left(\frac{1}{\theta_{0}}\right)^{k} V(0)+\sigma\|r(k)\|_{\infty}^{2} \sum_{j=0}^{k-1} \frac{1}{\theta_{0}^{k-j-1}} \\
& \leq \mu_{1}\left(\theta_{0}\right)\|\varphi\|_{\tau}^{2}+V(0)+\frac{\sigma}{\theta_{0}-1}\|r(k)\|_{\infty}^{2}, \forall k \geq 1 .
\end{aligned}
$$

On the other hand, set $\varpi=\alpha_{1}+\tau \alpha_{2}$, we can obtain

$$
V(0) \leq \varpi \sup _{j \in \mathbb{N}[-\tau, 0]}\|x(j)\|^{2} \text { and } V(k) \geq \lambda_{\min }(Q)\|x(k)\|^{2} .
$$

It follows that $\|y(k)\| \leq \theta_{1}\|r(k)\|_{\infty}+\theta_{2}, k \in \mathbb{N}^{+}$, where

$$
\theta_{1}=\|D\| \sqrt{\sigma\left(\theta_{0}-1\right)^{-1} \lambda_{\min }^{-1}(Q)}, \theta_{2}=\|D\| \cdot\|\varphi\|_{\tau} \sqrt{\left[\mu_{1}\left(\theta_{0}\right)+\varpi\right] \lambda_{\min }^{-1}(Q)} .
$$

By Definition 2, system (4) is BIBO stabilized by local control law (2), which complete the proof of Theorem 1.

Theorem 2 For given positive integer $\tau>0$, local control law (2) with feedback gain matrix $K$ stabilizes the delayed system (4), if there exist positive-definite matrices $Q, R, H, P_{1}, P_{2}$, positive-definite diagonal matrix $Z$ with appropriate dimensions, such that the following LMI holds:

$$
\Xi_{2}=\left[\begin{array}{ccccc}
\widetilde{\Xi}_{11} & \widetilde{\Xi}_{12} & \widetilde{\Xi}_{13} & \widetilde{\Xi}_{14} & 0 \\
* & \widetilde{\Xi}_{22} & \widetilde{\Xi}_{23} & \widetilde{\Xi}_{24} & 0 \\
* & * & \Xi_{33} & \Xi_{34} & \Xi_{35} \\
* & * & * & \Xi_{44} & \Xi_{45} \\
* & * & * & * & \Xi_{55}
\end{array}\right]<0,
$$

where $Q=\left[\begin{array}{ccc}Q_{11} & Q_{12} & Q_{13} \\ * & Q_{22} & Q_{23} \\ * & * & Q_{33}\end{array}\right]>0$

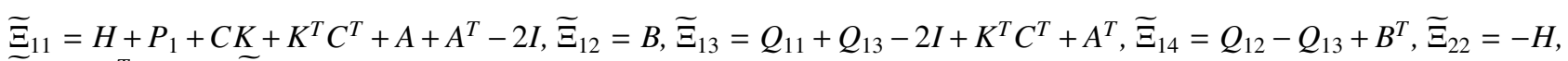
$\widetilde{\Xi}_{23}=Q_{12}^{T}+Q_{23}, \widetilde{\Xi}_{24}=Q_{22}-Q_{23}$.

Proof. Constructing augmented Lyapunov-Krasovskii function candidate as the same in Theorem 1. Set $\eta(k)=x(k+1)-$ $x(k)$, one can easily obtain this result, which omitted here.

Theorem 3 For given positive integer $\tau>0$, local control law (2) with feedback gain matrix $K$ robustly stabilizes the delayed system (4), if there exist positive-definite matrices $Q, R, H, P_{1}, P_{2}$, positive-definite diagonal matrix $Z$ with appropriate dimensions, and positive scalar $\epsilon>0$, such that the following LMI holds:

$$
\Xi_{3}=\left[\begin{array}{ccc}
\Xi_{2} & \xi_{1} & \epsilon \xi_{2}^{T} \\
* & -\epsilon I & 0 \\
* & * & -\epsilon I
\end{array}\right]<0
$$

where $Q=\left[\begin{array}{ccc}Q_{11} & Q_{12} & Q_{13} \\ * & Q_{22} & Q_{23} \\ * & * & Q_{33}\end{array}\right], \xi_{1}^{T}=\left[G^{T}, 0, G^{T}, 0,0\right], \xi_{2}=\left[E_{a}+E_{c} K, E_{b}, 0,0,0\right]$.

Proof. Replacing $A, B, C$ in inequality (18) with $A+G F(t) E_{a}, B+G F(t) E_{b}$ and $C+G F(t) E_{c}$, respectively. Inequality (18) for system (1) is equivalent to $\Xi_{2}+\xi_{1} F(t) \xi_{2}+\xi_{2}^{T} F^{T}(t) \xi_{1}^{T}<0$. From lemma 2 and lemma 3, one can easily obtain this result, which complete the proof.

Decomposing the weighting matrix $A$ as $A=A_{1}+A_{2}$. Set $\eta(k)=x(k+1)-A_{1} x(k)$, similar to the proof of Theorem 1 and Theorem 3, we can obtain the following less conservative criteria. 
Theorem 4 For given positive integer $\tau>0$, local control law (2) with feedback gain matrix $K$ stabilizes the delayed system (4), if there exist positive-definite matrices $Q, R, H, P_{1}, P_{2}$, positive-definite diagonal matrix $Z$ with appropriate dimensions, such that the following LMI holds:

$$
\Xi_{4}=\left[\begin{array}{ccccc}
\Xi_{11}^{\prime} & \Xi_{12}^{\prime} & \Xi_{13}^{\prime} & \Xi_{14}^{\prime} & \Xi_{15}^{\prime} \\
* & \Xi_{22}^{\prime} & \Xi_{23}^{\prime} & \Xi_{24}^{\prime} & \Xi_{25}^{\prime} \\
* & * & \Xi_{33} & \Xi_{34} & \Xi_{35} \\
* & * & * & \Xi_{44} & \Xi_{45} \\
* & * & * & * & \Xi_{55}
\end{array}\right]<0
$$

where $Q=\left[\begin{array}{ccc}Q_{11} & Q_{12} & Q_{13} \\ * & Q_{22} & Q_{23} \\ * & * & Q_{33}\end{array}\right]>0$,

$\Xi_{11}^{\prime}=A_{1}^{T} Q_{11} A_{1}-Q_{11}+H+P_{1}+C K+K^{T} C^{T}+A_{2}^{T}+A_{2}-2 I, \Xi_{12}^{\prime}=A_{1}^{T} Q_{12} A_{1}-Q_{12}+B, \Xi_{13}^{\prime}=A_{1}^{T}\left(Q_{11}+Q_{13}\right)-2 I+K^{T} C^{T}+A_{2}^{T}$, $\Xi_{14}^{\prime}=A_{1}^{T}\left(Q_{12}-Q_{13}\right)+B^{T}, \Xi_{15}^{\prime}=A_{1}^{T} Q_{13}-Q_{13}, \Xi_{22}^{\prime}=A_{1}^{T} Q_{22} A_{1}-Q_{22}-H, \Xi_{23}^{\prime}=A_{1}^{T}\left(Q_{12}^{T}+Q_{23}\right), \Xi_{24}^{\prime}=A_{1}^{T} Q_{22}-A_{1}^{T} Q_{23}$, $\Xi_{25}^{\prime}=A_{1}^{T} Q_{23}-Q_{23}$.

Theorem 5 For given positive integer $\tau>0$, local control law (2) with feedback gain matrix $K$ robustly stabilizes the delayed system (4), if there exist positive-definite matrices $Q, R, H, P_{1}, P_{2}$, positive-definite diagonal matrix $Z$ with appropriate dimensions, and positive scalar $\epsilon>0$, such that the following LMI holds:

$$
\Xi_{5}=\left[\begin{array}{ccc}
\Xi_{4} & \xi_{1} & \epsilon \xi_{2}^{T} \\
* & -\epsilon I & 0 \\
* & * & -\epsilon I
\end{array}\right]<0
$$

where $Q=\left[\begin{array}{ccc}Q_{11} & Q_{12} & Q_{13} \\ * & Q_{22} & Q_{23} \\ * & * & Q_{33}\end{array}\right], \xi_{1}^{T}=\left[G^{T}, 0, G^{T}, 0,0\right], \xi_{2}=\left[E_{a}+E_{c} K, E_{b}, 0,0,0\right]$.

\section{Numerical examples}

In this section, two numerical examples will be presented to show the validity of the main results derived above.

Example 1. Consider the delayed discrete-time system in (3) with parameters given by

$$
C=\left[\begin{array}{ll}
1.7 & 1.3 \\
0.3 & 1.6
\end{array}\right], A=\left[\begin{array}{ll}
0.5 & 0.0 \\
0.0 & 0.4
\end{array}\right], B=\left[\begin{array}{cc}
1 & 0.1 \\
0.2 & 0.1
\end{array}\right], \tau=3, A_{1}=A_{2}=0.5 A .
$$

One can check that LMI (5) in Theorem 1, LMI (18) in Theorem 2 and LMI (20) in Theorem 4 are feasible. By the Matlab LMI Toolbox, a feasible solution to the LMI (5) is obtained as follows:

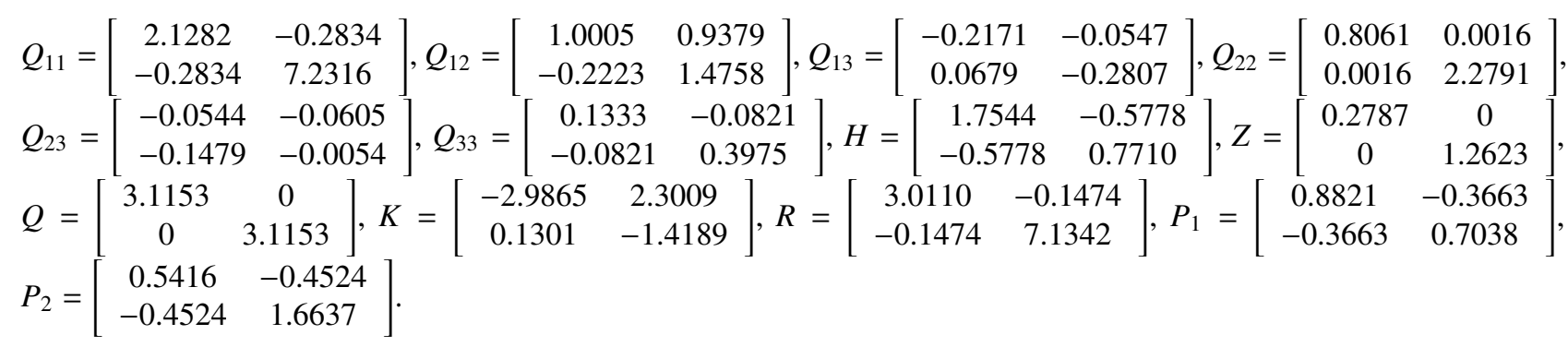

Example 2. Consider a delayed discrete-time system in (1) with parameters given by

$$
E_{a}=\left[\begin{array}{cc}
0.01 & 0 \\
0 & 0.12
\end{array}\right], E_{b}=\left[\begin{array}{cc}
0.03 & 0.1 \\
0.0 & 0.1
\end{array}\right], E_{c}=\left[\begin{array}{cc}
0.02 & 0.00 \\
0.00 & 0.02
\end{array}\right], G=\left[\begin{array}{cc}
0.02 & 0.0 \\
0.0 & 0.03
\end{array}\right],
$$

$A, B, C, A_{1}, A_{2}, \tau$ are the same as given in Example 1. One can check that LMI (19) in Theorem 3 and LMI (21) in Theorem 5 are feasible. By the Matlab LMI Toolbox, a feasible solution to the LMI (19) is obtained as follows:

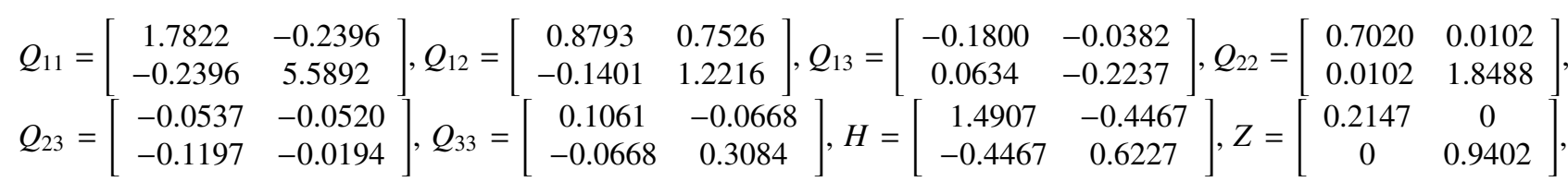


$\begin{aligned} Q & =\left[\begin{array}{cc}2.6640 & 0 \\ 0 & 2.6640\end{array}\right], K=\left[\begin{array}{cc}-2.5494 & 1.8497 \\ 0.1464 & -1.2458\end{array}\right], R=\left[\begin{array}{cc}2.4877 & -0.1559 \\ -0.1559 & 5.5087\end{array}\right], P_{1}=\left[\begin{array}{cc}0.7114 & -0.2956 \\ -0.2956 & 0.5605\end{array}\right], \\ P_{2} & =\left[\begin{array}{cc}0.4348 & -0.3614 \\ -0.3614 & 1.2885\end{array}\right] .\end{aligned}$

\section{Conclusion}

Combined with linear matrix inequality (LMI) technique, the problem of BIBO stabilization for a class of discrete-time delayed control system is investigated. By constructing an augmented Lyapunov-Krasovskii function, some new delaydependent conditions ensuring BIBO stabilization and robust BIBO stabilization are obtained. Numerical examples show that the new results are valid.

\section{References}

Bose. T., Chen. M. (1995). BIBO stability of discrete bilinear system. Digital Signal Processing, 5, 160-165.

Boyd. B. et al. (1994). Linear matrix inequalities in systems and control theory, Philadelphia (PA): SIAM.

Fu. Y, Liao. X. (2003). BIBO Stabilization of Stochastic Delay Systems With Uncertainty. IEEE Transactions on Automatic Control, 48, 133-138.

Guan. Z. et al. (1994). Variation of the parameters formula and the problem of BIBO for singular measure differential systems with impulse effect. Applied Mathematics and Computation, 60, 153-169.

Huang. Y, Zeng. W and Zhong. S. (2005). BIBO stability of continuous time systems. Journal of Electronic Science and Technology of China, 3, 178-181.

Kotsios. S., Feely. O. (1998). A BIBO stability theorem for a two-dimensional feedback discrete system with discontinuities. Journal of Frankhn Institute, 3358, 533-537.

Lee. T., Radovic. U. (1987). General decentralized stabilization of large-scale linear continuous and discrete time-delay systems. International Journal of Control, 46, 2127-2140.

Li. P. et al. (2007). Delay-dependent robust BIBO stabilization ..., Chaos, Solitons and Fractals. doi:10.1016/j.chaos. 2007.08.059.

Li. P, Zhong. S. (2008a). BIBO stabilization of time-delayed system with nonlinear perturbation. Applied Mathematics and Computation, 195, 264-269.

Li. P, Zhong. S. (2008b). BIBO stabilization for system with multiple mixed delays and nonlinear perturbations. Applied Mathematics and Computation, 196, 207-213.

Li. P, Zhong. S. M. (2009). BIBO stabilization of piecewise switched linear systems with delays and nonlinear perturbations. Appl. Math. Comput., doi: 10.1016/j.amc. 2009.03.029.

Liu. Y, Wang. Z and Liu. X. (2008). Robust stability of discrete-time stochastic neural networks with time-varying delays. Neurocomputing, 71, 823-833.

Michaletzky. G., Gerencser. L. (2002). BIBO stability of linear switching systems. IEEE Transactions on Automatic Control, 47, 1895-1898.

Partington. R., Bonnet. C. (2004). $H_{\infty}$ and BIBO stabilization of delay systems of neutral type. Systerm Control Letter, 52, 283-288.

Wolfgang. F, Mecklenbräuker. (1998). On the localization of impulse responses of BIBO-stable LSI-systems. Signal Processing, 70,73-74.

Xu. D., Zhong. S. (1995). The BIBO stabilization of multivariable feedback systems. Journal of Electronic Science and Technology of China, 24, 90-96. 